\title{
A Case of Subarachnoid Hemorrhage from the Posterior Pial Artery of the Medulla Oblongata
}

\author{
Takahiro Ogishima, MD, PhD ${ }^{1,2^{*}}$, Takashi Sugawara, MD, PhD ${ }^{1}$, Shoko Hara, $M D^{1}$, Tadashi Nariai, MD, \\ $P h D^{1}$, and Taketoshi Maehara, MD, PhD \\ ${ }^{1}$ Department of Neurosurgery, Tokyo Medical and Dental University, Tokyo, Japan \\ ${ }^{2}$ Department of Neurosurgery, Red Cross Musashino Hospital, Tokyo, Japan

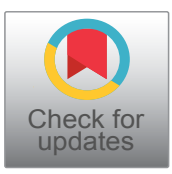

*Corresponding author: Takahiro Ogishima, MD, PhD, Department of Neurosurgery, Tokyo Medical and Dental University, Tokyo; Department of Neurosurgery, Red Cross Musashino Hospital, 1-26-1 Kyounancyho, Musashino-City, Tokyo 180-8610, Japan, Tel: +81-422-32-3111, Fax: +81-422-32-9551

\begin{abstract}
We present a case with localized subarachnoid hemorrhage in cisterna magna. The patient exhibited re-bleeding after admission. Repeated angiography couldn't find the bleeding cite. Surgical exploration revealed that the responsible artery was a tiny pial artery on the posterior surface of medulla oblongata. Even though the artery was too small to be delineated in angiogram, subarachnoid hemorrhage and re-bleeding did occur. In this report we discuss the cause of the small artery rupture and possible diagnostic evaluation, treatment. Although it is extremely rare, we should take into consideration the possibility of SAH from a pial artery.
\end{abstract}

\section{Keywords}

Subarachnoid hemorrhage, Pial artery, Medulla oblongata, Posterior inferior cerebellar artery occlusion, Hemodynamic stress, Super-selective angiography

\section{Abbreviations}

SAH: Subarachnoid hemorrhage; CT: Computerized tomography; VA: Vertebral artery; PICA: Posterior inferior cerebellar artery; ICG: Indocyanine green; PLBA: Primitive lateral basilo-vertebral anastomosis; LSA: Lateral spinal artery; 3DRA: Three dimensional rotational angiography; DSA: Digital subtraction angiography; CBCT: Cone-beam CT

\section{Introduction}

Subarachnoid hemorrhage (SAH) is a severe disease with high mortality and many of survivors will be left with disability. A key to good prognosis for cases with $\mathrm{SAH}$ is prompt diagnosis of the cause of bleeding and hemostasis to avoid re-bleeding that carries an even greater mortality rate. Although extremely rare, SAH does occur due to small arterial rupture. The diagnosis is quite difficult because of the small size of the artery. In this report, we describe a case of subarachnoid hemorrhage from the posterior pial artery of the medulla oblongata. That was related to hemodynamic stress caused by parent artery occlusion. Preoperative accurate diagnosis was difficult, but repeated and super-selective angiography including super-selective angiography provided important information that was revealed in surgical exploration.

\section{Case Report}

\section{History}

A 66-year-old female was transported to our hospital by an ambulance with complaints of sudden posterior cervical pain and vomiting. She had a history of hypertension and SAH due to aneurysmal rupture of the distal anterior cerebral artery 9 years ago. She had undergone aneurysmal neck clipping surgery and fully recovered. On arrival, she was alert, had a stiff neck, posterior cervical pain, and nausea, but no other focal symptoms. A computerized tomography scan (CT scan) revealed SAH with thick hematoma in the cisterna magna spreading into the ventricles, towards the foramen Monro (Figure 1). The CT angiography also revealed a saccular aneurysm of $3 \mathrm{~mm}$ diameter at the bifurcation of the internal

Citation: Ogishima T, Sugawara T, Hara S, Nariai T, Maehara T (2020) A Case of Subarachnoid Hemorrhage from the Posterior Pial Artery of the Medulla Oblongata. Neurosurg Cases Rev 3:044. doi. org/10.23937/2643-4474/1710044

Accepted: October 27, 2020; Published: October 29, 2020

Copyright: (c) 2020 Ogishima T, et al. This is an open-access article distributed under the terms of the Creative Commons Attribution License, which permits unrestricted use, distribution, and reproduction in any medium, provided the original author and source are credited. 

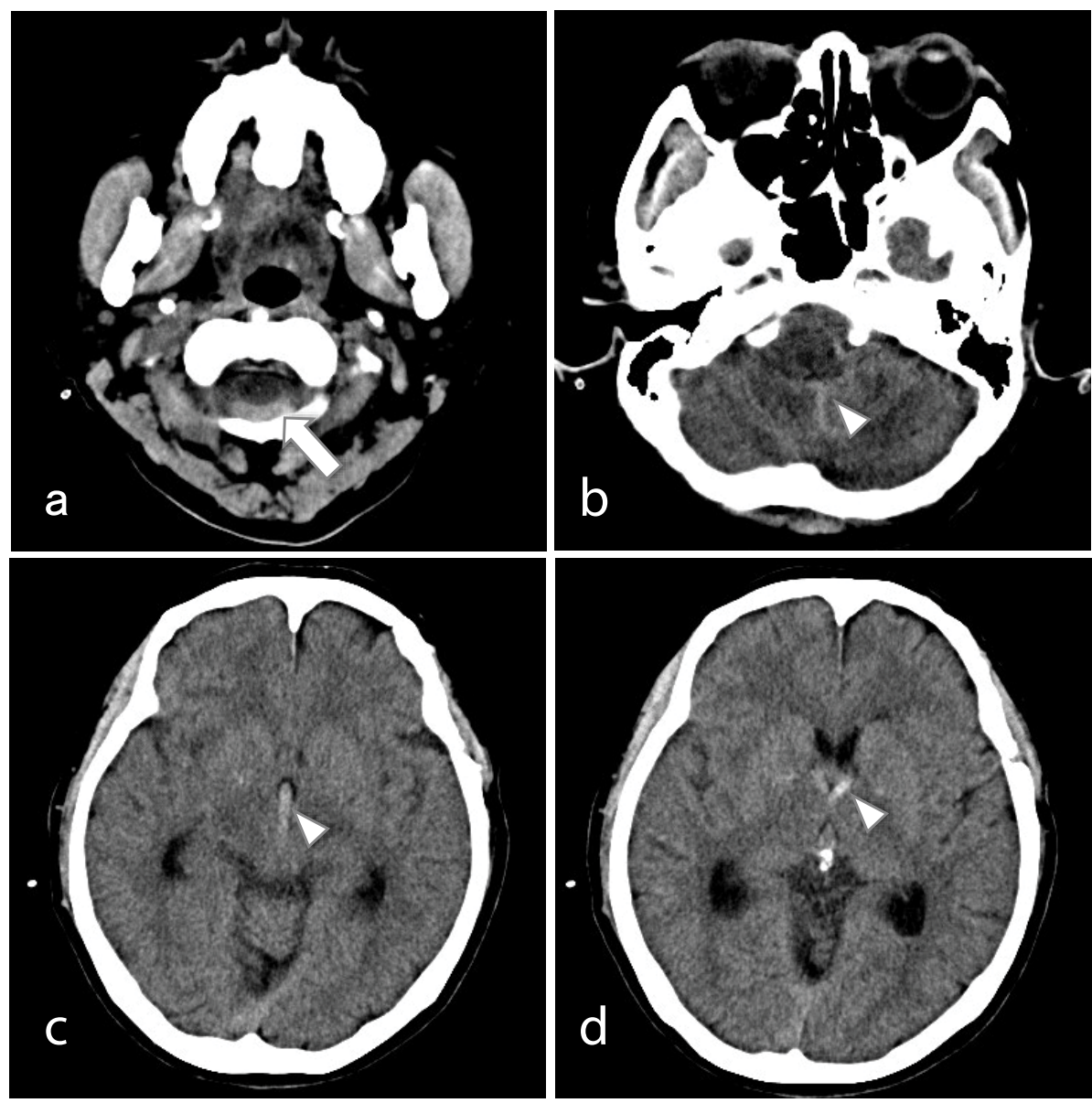

Figure 1: (a: Arrow) CT scan on arrival show thick hematoma in cisterna magna; (b-d: Arrow heads) The hematoma spread to ventricles and subsequently to foramen Monro.
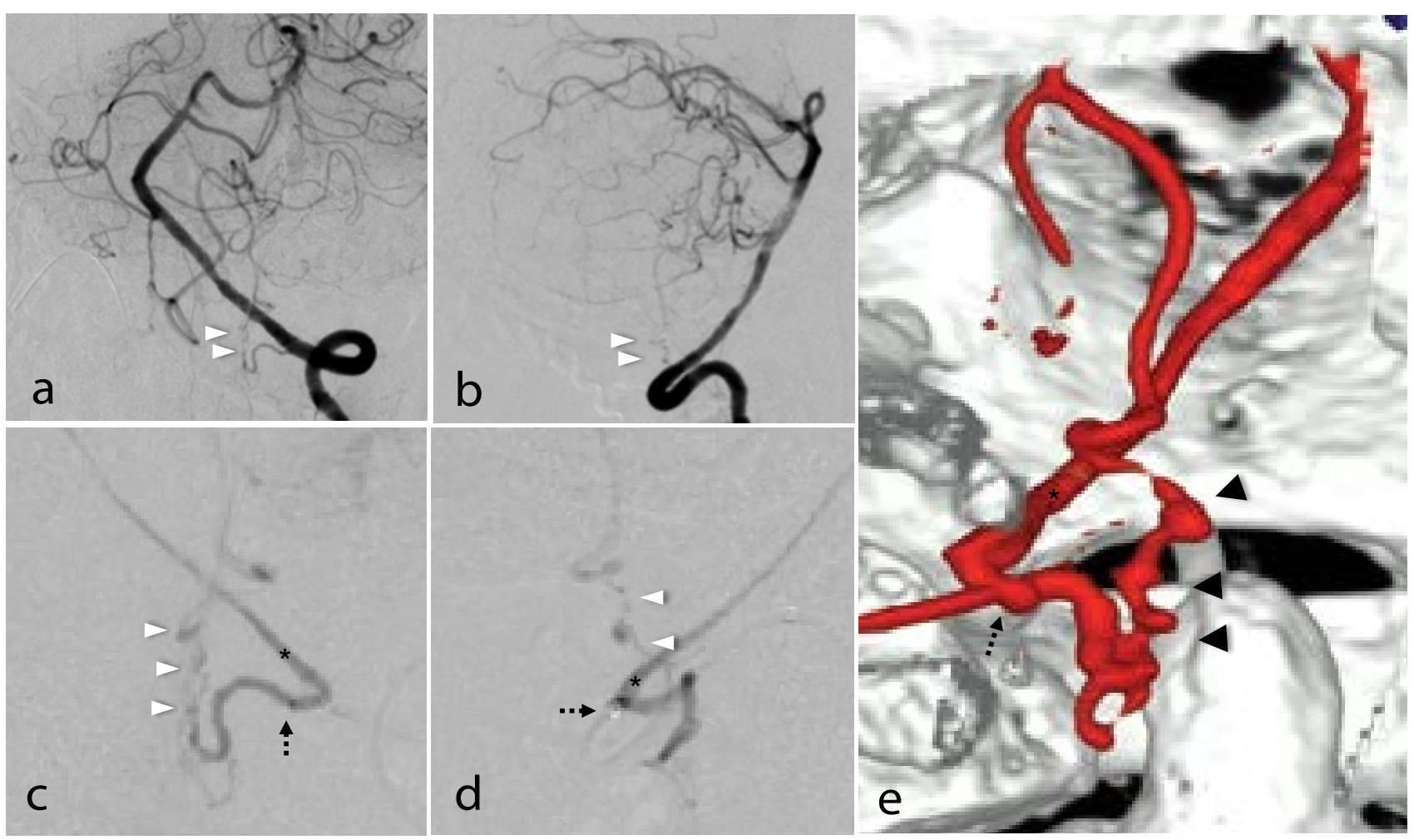

Figure 2: Angiography (a: A-P view, b: Lateral view) Super-selective angiography of left PICA; (c: A-P view, d: Lateral view, e: 3D-DSA). The tip of microcatheter was at the origin of the left PICA (dotted arrow). Left VA was partially visualized (asterisk in 2c-2e). Irregularity of the small artery (arrow heads) was shown. 
carotid artery and the posterior communicating artery, but no other vascular abnormality in posterior circulation. A cerebral angiography was also done but added no more information. A conservative treatment approach with antihypertensive drugs and bed rest were initiated. On the same day after the admission, she still showed signs of repeated vomiting and the CT scan revealed the hematoma growth. She was intubated and kept deeply sedated to prevent further bleeding. Another cerebral angiography including super-selective angiography was taken three days after the admission, which revealed irregularity of a small artery branching from the left vertebral artery (VA) (Figure 2). We assumed the artery to be the early branching left posterior inferior cerebellar artery (PICA) as it ascended from the left VA to perfuse the lower area of the cerebellum distally, and the irregularity was due to dissection of the artery. The anatomical location of the left PICA was just in the place where the thick hematoma was. Although it was difficult to make an accurate diagnosis at that time because the artery was too small to be delineated precisely even on the super selective angiograph, we suspected the culprit artery for the SAH was the dissecting left PICA. Endovascular surgery was difficult because the artery was too small. We planned the open surgery four days after the admission in order to prevent further bleeding and to make definite diagnosis.

\section{Operation}

The patient underwent surgery by lateral suboccipital approach with $\mathrm{C} 1$ hemi laminectomy. Thick hematoma was seen on the posterior surface of medulla oblongata. The hematoma was thick and localized so that the bleeding point was presumed to be just under the hematoma (Figure 3). After the hematoma was removed, bleeding point in a pial artery was revealed. Weak arterial bleeding was seen in the medullary pial artery after removing the hematoma. The bleeding point (arrow in Figure 3) was not in the left PICA was rather found in the medullary pial artery (arrow heads in Figure 3 ) that originated from the proximal portion of the left PICA.
The artery merged into the distal portion of the left PICA distally. Also, the occlusion of the left PICA just distal to the branching point of the medullary pial artery was revealed. The medullary pial artery formed the collateral pathway. The artery was slightly dilated near the bleeding point (dotted arrow in Figure 3). Confirming that the PICA distal to the occluded lesion was visualized by indocyanine green (ICG) in a retrograde fashion, the bleeding point was carefully coagulated using bipolar forceps in order to arrest bleeding. Because we had suspected the dissecting PICA was the culprit artery for the $\mathrm{SAH}$, we decided to examine the pathology of the lesion of PICA. The occluded lesion of the PICA was trapped with proximal as well as distal clipping and resected to pathological examination.

Pathological findings of the resected PICA revealed the hemosiderosis and fibrosis in the media, without apparent dissection.

\section{Postoperative course}

Postoperative course was favorable without severe complication. The patient was shifted to a suburban hospital for rehabilitation treatment at 48 days after the onset. At follow-up around 3 months after the onset, the patient had no neurological deficit.

\section{Discussion}

\section{Cause of the small artery rupture}

This is an extremely rare case of SAH from the medullary pial artery. Although the bleeding point was slightly dilated, the change was too small to be delineated in the pre-surgical angiography. We assumed that the cause of SAH was related to the vulnerability of the small artery and hemodynamic change due to PICA occlusion.

Anatomically, arteries of the posterior surface of the upper cervical spinal cord and the medulla oblongata tend to make small arterial plexus contrary to the artery of anterior surface. These arteries develop from the primitive lateral basilo-vertebral anastomosis (PLBA)

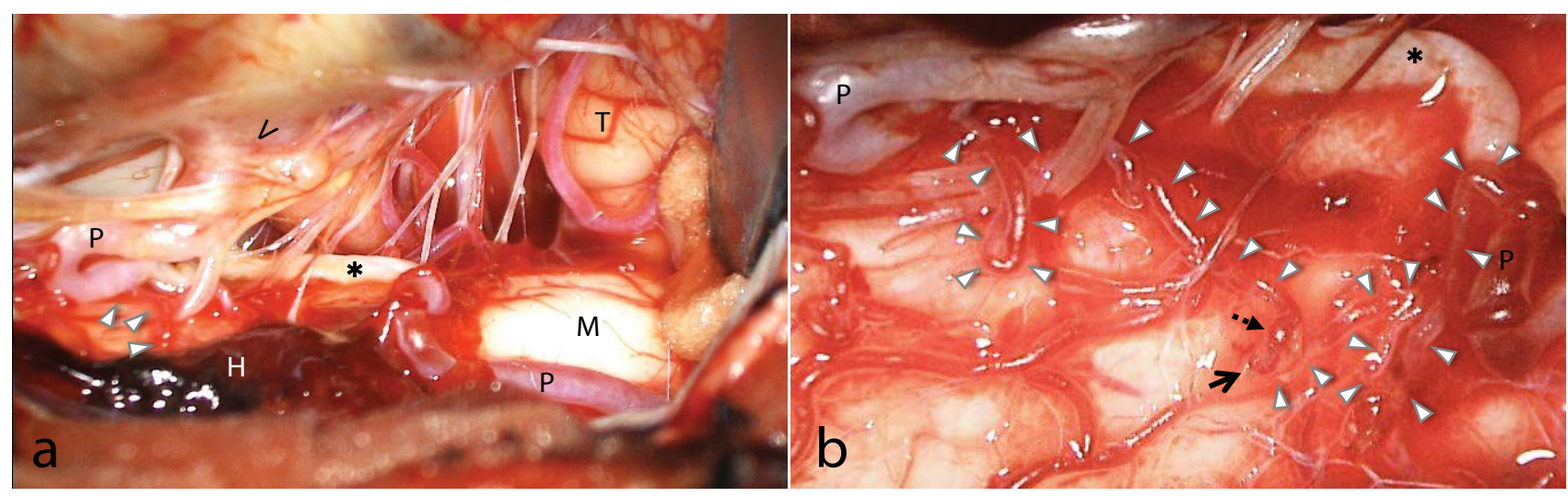

Figure 3: Intraoperative photographs (a) Thick hematoma was seen on the posterior surface of medulla oblongata; (b) After the hematoma was removed, bleeding point in a pial artery was revealed.

H: Hematoma, M: Medulla oblongata, P: PICA, T: Tonsil, V: VA, Asterisk: occluded PICA. 
referred by Padget, and could have anastomosis with PICA or VA that have the same origin. In our case, the responsible artery coursed medially and rostrally, posterior to the $11^{\text {th }}$ nerve rootlet, making plexus of small arteries on the posterior surface of the medulla oblongata. It coursed more on the medial and dorsal side, but developmentally similar to lateral spinal artery (LSA). Chen, et al. reported two cases with saccular aneurysms of the LSA related to the high-grade stenosis of the parent artery [1]. They noted that the increased hemodynamic stress from the collateralization due to the severe stenosis of parent artery likely contributed to the aneurysm formation and rupture. Although it is rare, there have been a few reported cases of aneurysm of the LSA related to the same mechanism of hemodynamic stress [1-3].

The characteristic finding in our case was that there was no apparent change on the response artery other than slight dilatation of the bleeding point. In the operative findings, we could not find an enlargement or any other morphological changes on the artery compared with the neighboring pial arteries (Figure 3 ). That was the reasons why preoperative accurate diagnosis was quite difficult. This might have resulted from the duration of hemodynamic stress and the size of the responsible artery. Recent studies showed that chronic inflammation and degradation of the extracellular matrix in the vessel wall induced by long term hemodynamic stress are associated with the formation of the aneurysm $[4,5]$. It was also suggested that it takes a few weeks to detect an experimentally induced cerebral aneurysms in rats after the aneurysmal induction. Given the situation that hemodynamic stress occur on the vulnerable artery in the short term, collapse of the vessel wall without aneurysmal formation or other morphological change may happen.

\section{Diagnostic evaluation, treatment}

In this case, it was difficult to make accurate diagnosis before the operation in spite of the repeated cerebral angiography. The main reason for the failure in diagnosis was the size of the responsible artery. We assumed the dissection of the left PICA. The fact was the pial artery rupture with occlusion of PICA. The micro-surgical findings revealed that what we thought as dissecting lesion was an almost normal pial artery.

In general, approximately $10-20 \%$ of spontaneous SAH is angiogram-negative [6-8]. Tatter, et al. retrospectively reviewed seven patients with angiogram-negative SAH who had micro aneurysm discovered in the surgical exploration [9]. They pointed out that these micro aneurysms were too small to delineate even in ideally oriented oblique images. Along with the development of the instrument, rates of angiogram-negative $\mathrm{SAH}$ were diminished. Ishihara, et al. reported the efficacy and superiority of three dimensional rotational angiography
(3DRA) compared with the classical digital subtraction angiography (DSA), especially for aneurysms with less than $4 \mathrm{~mm}$ diameter [10]. However, they admitted that $4.2 \%$ of spontaneous SAH was still angiogram-negative even using 3DRA. Super-selective angiography could add information about location of bleeding point, and helped to prevent surgical complications. Recently, there were some reports about the benefit of conebeam CT (CBCT) angiography for detecting precise information on the cerebral vascular lesions $[11,12]$. Repeated and super-selective angiography in combination with such high-resolution studies might be useful for searching such tiny vessel lesions.

In terms of treatment, surgical exploration and if possible, clipping (or trapping, wrapping) in acute stage should be utilized for the purpose of diagnosis and hemostasis. Because of the small diametric size of the vessels and difficulty in diagnosis, endovascular approach may not be suitable. Angiogram-negative SAH other than perimesencephalic type has relatively high risk of re-bleeding and is not necessarily a good outcome [68,13-15]. Re-bleeding in acute phase could occur even if the size of the responsible artery and aneurysm was very small like this case. We recommend that if the hematoma is localized, surgical exploration in acute stage seems to be reasonable considering the risks and benefits involved.

\section{Conclusion}

We report an extremely rare case of SAH from medullary pial artery. Detecting the bleeding point is quite difficult preoperatively. However, re-bleeding could occur even the small arterial rupture. This necessitates the need for repeated angiography in combination with super-selective angiography and prompt surgical exploration. Newly introduced high-resolution studies such as CBCT angiography might be useful in these small lesions.

\section{Ethics}

The patient provided her informed consent for the inclusion of her clinical data in this manuscript.

\section{Disclosure}

The authors declare that they have no conflict of interest concerning the materials or methods used in this paper.

\section{Author Contribution}

Ogishima, Sugawara, and Hara carried out the surgical treatment and clinical management of the patient. Ogishima wrote the manuscript with support from Sugawara, Hara, Nariai, and Maehara. Nariai and Maehara supervised the findings of this work. All authors discussed the results and contributed to the final manuscript. 


\section{References}

1. Chen CC, Bellon RJ, Ogilvy CS, Putnam CM (2001) Aneurysms of the lateral spinal artery: Report of two cases. Neurosurgery 48: 949-953.

2. Kubota H, Suehiro E, Yoneda H, Nomura S, Kajiwara K, et al. (2006) Lateral spinal artery aneurysm associated with a posterior inferior cerebellar artery main trunk occlusion Case illustration. J Neurosurg Spine 4: 347.

3. Morigaki R, Satomi J, Shikata E, Nagahiro S (2012) Aneurysm of the lateral spinal artery: A case report. Clin Neurol Neurosurg 114: 713-716.

4. Aoki T, Kataoka H, Ishibashi R, Nozaki K, Egashira K, et al. (2009) Impact of monocyte chemoattractant protein-1 deficiency on cerebral aneurysm formation. Stroke 40: 942-951.

5. Aoki T, Kataoka H, Shimamura M, Nakagami H, Wakayama K, et al. (2007) NF-kappaB is a key mediator of cerebral aneurysm formation. Circulation 116: 2830-2840.

6. Fontanella M, Rainero I, Panciani PP, Schatlo B, Benevello $C$, et al. (2011) Subarachnoid hemorrhage and negative angiography: Clinical course and long-term follow-up. Neurosurg Rev 34: 477-484.

7. Rinkel GJE, Vangijn J, Wijdicks EFM (1993) Subarachnoid hemorrhage without detectable aneurysm - A review of the causes. Stroke 24: 1403-1409.

8. Rinkel GJE, Wijdicks EFM, Hasan D, Kienstra GEM, Franke CL, et al. (1991) Outcome in patients with subarachnoid hemorrhage and negative angiography according to pattern of hemorrhage on computed-tomography. Lancet 338: 964968.
9. Tatter SB, Crowell RM, Ogilvy CS (1995) Aneurysmal and microaneurysmal angiogram-negative subarachnoid hemorrhage. Neurosurgery 37: 48-55.

10. Ishihara H, Kato S, Akimura T, Suehiro E, Oku T, et al. (2007) Angiogram-negative subarachnoid hemorrhage in the era of three dimensional rotational angiography. J Clin Neurosci 14: 252-255.

11. Lauric A, Heller RS, Schimansky S, Malek AM (2015) Benefit of cone-beam CT angiography in visualizing aneurysm shape and identification of exact rupture site. J Neuroimaging 25: 56-61.

12. Rahal JP, Malek AM (2013) Benefit of cone-beam computed tomography angiography in acute management of angiographically undetectable ruptured arteriovenous malformations Clinical article. J Neurosurg 119: 1015-1020.

13. Boswell S, Thorell W, Gogela S, Lyden E, Surdell D (2013) Angiogram-negative subarachnoid hemorrhage: outcomes data and review of the literature. J Stroke Cerebrovasc Dis 22: $750-757$

14. Kostic A, Stojanov D, Stefanovic I, Novak V, Kostic E, et al. (2012) Complications after angiogram-negative subarachnoid haemorrhage: Comparative study of pretruncal and nonpretruncal hemorrhage patients. Srp Arh Celok Lek 140: 8-13.

15. Lin N, Zenonos G, Kim AH, Nalbach SV, Du R, et al. (2012) Angiogram-negative subarachnoid hemorrhage: relationship between bleeding pattern and clinical outcome. Neurocrit Care 16: 389-398. 